\title{
ON TWO-SCALE MODEL OF FRACTURE MESOMECHANICS OF COMPOSITES WITH CRACKS UNDER COMPRESSION
}

\author{
A.N.Guz \\ Institute of Mechanics, Nesterov str.3, 03680, Kiev, Ukraine
}

\section{ABSTRACT}

The two-level model of composites fracture with cracks under compression is proposed as applied to following problem. Consideration is given to fiber-laminated panels with hole compressed along the fibers so that two cracks propagate from hole edge in direction perpendicular to the loading axis. In above mentioned situation the analysis of fracture mechanism is presented under two-level consideration. First level of consideration corresponds to analysis of stress concentration near the crack tip under uniaxial compression as applied to the crack of finite thickness in case when the crack is filled by fractured material. At this stage the theory of elasticity of linear orthotropic body is used. Second level of consideration corresponds to analysis of fracture mechanism at the crack tip under compression taking into account the interface between fractured and intact materials. Threedimensional linearized theory of stability of solids is applied to analysis of the fracture mechanism at the crack tip taking into account the microcracks in the interface of fractured and intact materials. At this stage of investigation the exact solution of problem on instability of the interface with interfacial cracks in compression along interface is used. At the analysis on second level the authors results are used. Thus in the approach under consideration the methods of the theory of elasticity of linear orthotropic body and the methods of the three-dimensional linearized theory of stability of solids are applied to analysis of specific mechanism of fracture.

\section{INTRODUCTION}

Consideration is given to the laminated composite panels weakened by circular hole (Fig.1) in case of compression along the $0 y$-axis. The layers were stacked (along the $0 z$-axis) so that $0 x$ - and $0 y$-axis are the axes of symmetry of mechanical characteristics of the laminated composite materials, i.e., the composite can be considered as orthotropic material. Each layer of the material is an unidirectional fibrous composite, moreover the fibers in the majority of layers are also oriented along the $0 y$-axis (along the loading direction). Thus, the preferential reinforcement direction in the laminated composite plates under consideration is along the $0 y$-axis (Fig.1). It must be declared that the fracture theory for case under consideration (Fig.1) can't be developed on the base of Griffith-Irwin conception as this conception does not describe the fracture under 
compression. In view of its for case under consideration the fracture theory on the base of stability loss in internal structure of composite materials is proposed.

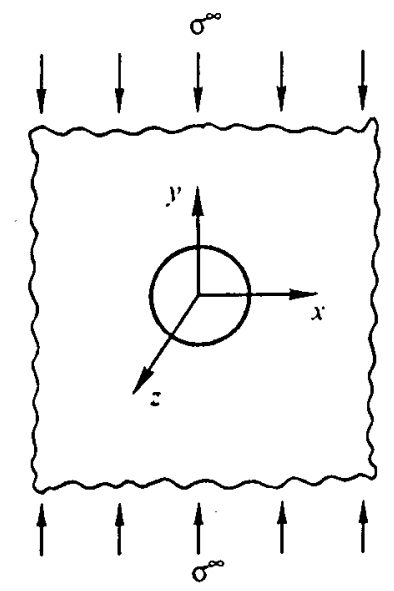

Figure 1: Panels weakened by hole in compression

\section{BODY OF PAPER}

In experimental investigations of some authors the following fracture mechanism was described. In all cases, the fracture started developing from zones with maximum of stress concentration (in Fig.1, from two point at the interaction of the horizontal axis and the contour of the hole). The observed fracture may be divided into two stage. First stage corresponds to the formation of two zones of fractured materials elongated along the $0 x$-axis at Fig.2. These zones of fractured material at Fig.2-5 are marked by the index "_." the intact material at Fig.2-5 is marked by index "+". It must be remarked the fractured zones are detected under loads that constitute up to $80-95 \%$ of the ultimate loads. The second stage corresponds to the beginning of the general fracture. This general fracture consists in the formation of macrocracks at points $A$ (Fig.2) propagating along $0 x$-axis (Fig.2), above mentioned macrocracks at points $A$ along $0 x$-axis are marked at Fig. 2 by bold line. In this paper the general fracture corresponds to the second stage.

The type of fracture under consideration may be characterized by following three basic features.

1. Even prior to general fracture (80-95\% of the ultimate load), comparatively narrow zones of fractured material arise and exist where the stress concentration is 
maximum (Fig.2, the zones with index “-“). These zones are strongly extended along the horizontal axis (along the $0 x$-axis at Fig.2).

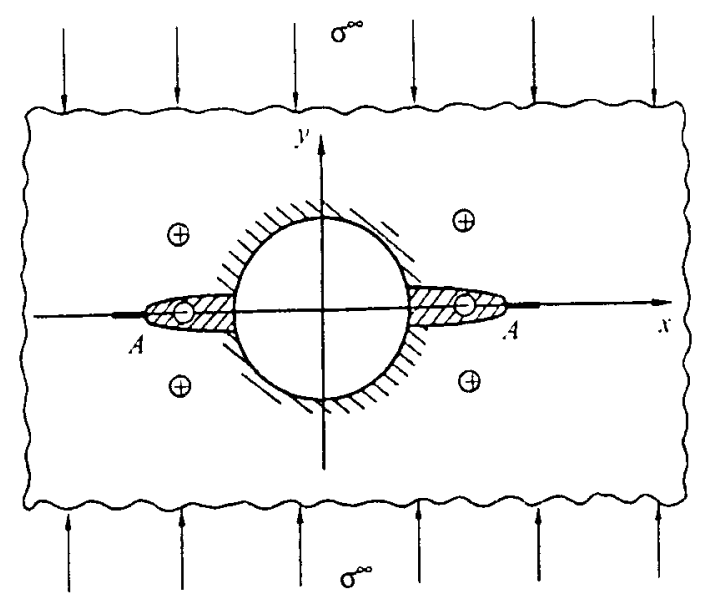

Figure 2: Scheme of fracture

2. These zones are finite in thickness (the size along the $0 y$-axis at Fig.2). The thickness of these zones is equal to several fibers diameters (no less two diameters), as one broken fiber (initially directed along $0 y$-axis at Fig.2) transforms into two fibers (two part of fiber) along $0 x$-axis.

3. The fracture zones are almost perpendicular to the direction of the compressive load, i.e. , the fracture may be said to propagate almost perpendicularly to the direction of the compressive load as applied to the second stage of fracture.

Above mentioned three basic features are taken into account in the two-scale model of fracture mesomechanics under consideration of composite panels with circular hole in compression.

First level of investigations in the framework of the two-level model under consideration corresponds to analysis of stress concentration near the crack tip in compression as applied to the elongated crack of finite thickness in case when the crack is filled by fractured materials. The above mentioned crack-tip vicinity is presented at Fig.3. At the first level of investigations the theory of elasticity of linear orthotropic body is used. Model of elongated elliptical hole filled by fractured material (Fig.4) is used in order to determine the stress concentration near crack-tip vicinity of zones of fractured material. In this case the semiaxes $a$ and $b$ of ellipse may be determined by special manner. 


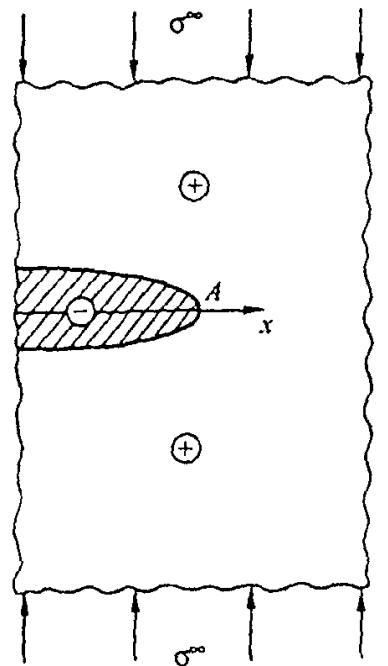

Figure 3: Crack-tip vicinity in case of filled crack

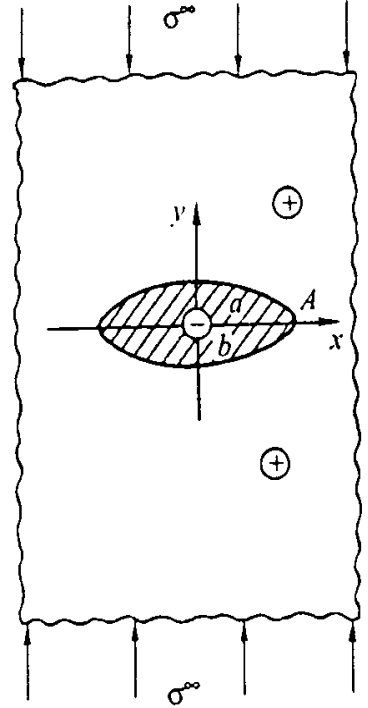

Figure 4: Model for determination of stress concentration

Second level of investigations in the framework of the two-level model under consideration corresponds to analysis of the fracture mechanism at point $A$ (Fig.2Fig.4). As it was remarked at INTRODUCTION in situation under consideration the $0 y$-axis (Fig.1-Fig.4) is the axis of the preferencial reinforcement of the laminated composite material, the axis of symmetry of mechanical characteristics of the laminated composite material and the axis of uniaxial compression. In this case the most probable mechanism of fracture start is mechanism of stability loss in structure of composite material.

Thus, the fracture mechanism being discussed and the approach to its study can be formulated as follows (as applied to second level of investigations in the framework of the two-level model under consideration).

The fracture start sets in the vicinity of point $A$ as local buckling described by the continuum theory of fracture of compressed composites and the basic relationships of the three-dimensional linearized theory of stability of solids (for an example, monograph [1]). Such an approach determines the theoretical ultimate compression strength and the direction of fracture propagation. 
This approach as applied to the internal fracture of composite materials in compression was proposed in [2] and was extended in [3] as applied to the nearthe-surface fracture of composite materials in compression near the concentrators of stresses. Above mentioned results were included in monograph [4].

Taking into account the proposed approach the vicinity of point $A$ (Fig.1-Fig.4) at the second level of investigation is modeled by two different half-planes with interfacial microcracks in compression (Fig.5).

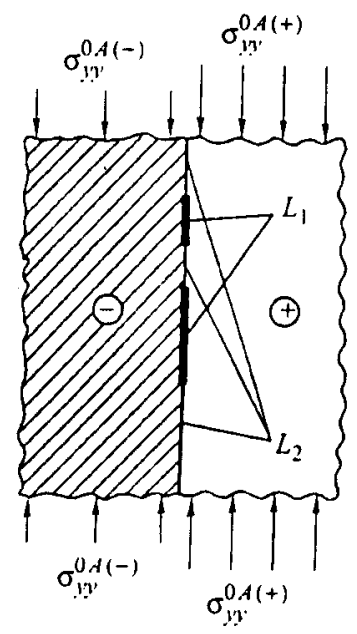

Figure 5: Model for fracture investigations of the vicinity of point $A$ at the second level of analysis

The compressive loads (Fig.5, $\sigma_{y y}^{O A(-)}$ - for fractured zone, $\sigma_{y y}^{O A(+)}-$ for intact zone) at point $A$ are determined under first level investigations of stress concentration problem. Exact solution of the problem presented at Fig.5 in the framework of the three-dimensional theory of stability of solids was published in $[5,6]$ and other publications cited in $[5,6]$. This exact solution $[5,6]$ is used for analysis of fracture under the second level of investigations.

It must be remarked the two-level model of fracture mesomechanics under consideration investigates the start of fracture at point $A$ only (beginning of fracture process), in this situation the most probable mechanism of fracture start is the stability loss in internal structure of composite material. In continuation of fracture process the stability loss in internal structure of composite material (microbuckling) arised at fracture start may be transformed into other mechanisms 
(delamination, shredding, end crushing, kinking, separation into isolated strands and fibers, etc.). Of course the above mentioned and other similar mechanisms are observed at the final stage of fracture process. In many cases the photographs of the fractured panels which are analysed and considerated by majority of the authors correspond to the final stage of fracture process. In view of it's the mechanisms (delamination, shredding, end crushing, kinking, separation into isolated strands and fibers, etc.) are fixed at the photographs.

\section{REFERENCES}

1. Guz, A.N., Fundamentals of the Three-Dimensional Theory of Stability of Deformable Bodies, Berlin, Springer-Verlag, 1999, 555 p.

2. Guz, A.N., On the determination of the theoretical strength limit in compression of the reinforced materials, Dopovidi Akademii Nauk Ukrain'skoj RSR, Ser.A, N 3, 236-238, 1969 (in Ukrainian).

3. Guz, A.N., On criterion of brittle fracture of the materials with defects in compression, Doklady Akademii Nauk SSSR, v.285, N 4, 828-831, 1985 (in Russian).

4. Guz, A.N., Fracture mechanics of composite materials under compression, Kiev, Naukova Dumka, 1990, 630 p. (in Russian).

5. Guz, I.A., Guz, A.N., Stability of two different half-planes in compression along interfacial cracks : Analytical solutions, Abstracts of XXth Int.Cong. of Theor. and Appl. Mech., Chicago, USA, Aug.27-Sep.2, 19-19, 2000.

6. Guz, I.A., Guz, A.N., Stability of two different half-planes in compression along interfacial cracks. Analytical solutions, Int. Appl. Mech., v.37, N 7, 906-912, 2001 